\title{
Video Watermarking for Copyright Protection with Genetic-Based Frame Selection
}

\author{
Saud S. Alotaibi \\ Department of Information Systems, College of Computer and Information Systems, Umm Al Qura \\ University, Makkah, Saudi Arabia \\ ssotaibi@uqu.edu.sa
}

\begin{abstract}
Recently, video watermarking has received much consideration. Several applications in a variety of domains have been implemented, and many are progressing. This paper intends to formulate a novel video watermarking framework that includes three stages: (i) Optimal video frame prediction, (ii) A watermark embedding process, and (iii) A watermark extraction process. In the proposed model, the optimal frame prediction is carried out using the deep belief network (DBN) framework. Initially, randomly chosen frames from each video are used as the input to a genetic algorithm (GA) model that optimally chooses the frames such that the peak signal-tonoise ratio (PSNR) should be maximal. The frames are assigned with a label of one or zero, where a label of one denotes a frame with better PSNR (can select for embedding process) and a label of zero denotes the frame with reduced PSNR (cannot be used for embedding). Consequently, a data library is formed from the obtained results, where each video frame is determined with their graylevel cooccurrence matrix (GLCM) features and labels (can embed or not), which is then trained in the DBN framework, from which the optimal frames can be predicted efficiently while testing. Furthermore, the watermark embedding process and watermark extraction process are carried out, and thus, the image can be embedded within the optimally selected frames.
\end{abstract}

Keywords: Video watermarking, Genetic algorithm, GLCM features, Deep belief network, Error measures.

$\begin{array}{ll}\text { Abbreviations } \\ \text { DBN } & \text { Deep Belief Network } \\ \text { GA } & \text { Genetic Algorithm } \\ \text { PSNR } & \text { Peak Signal-to-Noise Ratio } \\ \text { GLCM } & \text { Gray-Level Cooccurrence Matrix } \\ \text { IPR } & \text { Intellectual Property Rights } \\ \text { DWT } & \text { Discrete Wavelet Transform } \\ \text { BWT } & \text { Biorthogonal Wavelet Transform } \\ \text { ABC } & \text { Artificial Bee Colony } \\ \text { SIFT } & \text { Scale Invariant Feature Transform } \\ \text { BE } & \text { Blind Extraction } \\ \text { HEVC } & \text { High-Efficiency Video Coding } \\ \text { VBR } & \text { Video Bit Rate } \\ \text { MSE } & \text { Mean Squared Error } \\ \text { LSB } & \text { Least Significant Bit } \\ \text { SDME } & \text { Second Derivative-like Measure of Enhancement }\end{array}$

\section{Introduction}

In recent years, the privacy of important documents has become essential. In the fastgrowing computer environment, maintaining the secrecy of soft copies of medical records is a major challenge and becomes even more difficult in hospitals as the number of patients increases ${ }^{[1-3]}$. Hence, a proper and suitable application is essential to secure the required data. Watermarking has attracted considerable attention recently [4-6], and it provides protection to digital content and conserves the 
copyright protection of video or audio images. Furthermore, digital watermarking has been regarded as a proper solution for protecting copyrights and implementing IPR ${ }^{[7-9]}$. The digital manuscript could be video, image, audio or text. If the digital manuscript is in video signal form, then the embedding method is termed video watermarking ${ }^{[10-12]}$. It was moreover known as an imperceptible and robust form of digital video data communication.

If watermarking requires the original video signal to be available at the watermark detector, then it enables informed detection. Additionally, a watermarking approach employs blind detection ${ }^{[13-15]}$ if the original video signal need not be available at the watermark detector. Watermarking schemes have to own particular aspects on the basis of the watermark type and its purpose. In authentication applications, digital watermarks can be used to detect modifications of the digital contents (e.g., compression). Content authentication is an important application of video watermarking. In addition, multimedia copyright protection of video signals is protected with the help of digital watermarking based on the DWT model ${ }^{[16,17]}$. Furthermore, robustness can be enhanced with the transform domain video watermarking, in which the video signal is transformed into a lowfrequency domain. Thus, video watermarking remains a prospective scheme for protecting copyrights ${ }^{[18,19]}$.

The major contribution of this paper is depicted below.

1. Contributing a new video watermarking framework that initially determines which frames are optimal for performing the embedding process. This optimal selection of frames is carried out via a genetic algorithm.
2. Creating a data library that insists whether the frame is better for embedding purposes along with extracting GLCM features.

3. Furthermore, DBN is trained with the details of the data library and helps in predicting the optimal frames at the time of the testing process.

4. Finally, watermark embedding and extraction are performed.

The arrangement of the paper is as follows. Section 2 analyzes the literature work. Section 3 describes the proposed video watermarking framework, and Section 4 portrays the video watermark embedding and watermark extraction process. Further, Section 5 illustrates the results, and Section 6 concludes the paper.

\section{Literature Review}

\section{A. Related Works}

In 2018, Sake and Ramashri ${ }^{[1]}$ presented a video watermarking technique that deploys the BWT approach for protecting the copyrights of images. To enhance the effectiveness of video watermarking, two major processes were exploited: watermark extraction and watermark embedding. Prior to the embedding method, the sequences of input video are transformed into numerous frames. In addition, an enhanced ABC model was introduced for producing arbitrary frames. Subsequently, a watermark extraction process was performed, which is the inverse procedure of watermark embedding.

In 2017, Sahu and Arijit ${ }^{[2]}$ implemented a blind video watermarking model that avoids frame adaptation and frame dropping owing to the SIFT. In video scenery, counts of rows were assigned based on height and count of frames were assigned based on width and count of columns were assigned based on depth. In this context, the SIFT features were exploited 
for the watermark signal and were stored for authentication purpose. Finally, a wide-ranging set of experiments were performed to demonstrate the effectiveness of the implemented method over other conventional schemes.

In 2016, Tanima and Hari ${ }^{[3]}$ suggested a robust structure with a $\mathrm{BE}$ procedure for HEVC encoded videos. A "readable watermark" was incorporated in the predicted video blocks sequence, and moreover, the presented scheme offered better security in the embedding regions. The investigational outcomes revealed that the introduced work limits the increase in the VBR, and it was also found to improve quality. Thus, the introduced scheme could prevail over compressions, filtering, and noise constraints, thereby offering superior robustness and quality.

In 2016, Rasti et al. ${ }^{[4]}$ presented a robust nonblind color video watermarking approach. Initially, the technique split the frames into nonmoving and moving elements, and further, the nonmoving elements of every color channel were separately processed by means of a blockoriented watermarking system. Blocks with lower entropy were subjected to another process for watermark image embedding, and thus, the watermarked frame could be produced. In addition, numerous attacks were analyzed and distinguished with certain conventional techniques. Investigational outcomes demonstrate that the adopted method was robust against most of the attacks.

In 2018, Faride et al. ${ }^{[5]}$ established a novel blind multiplicative video watermarking approach. Here, the $\mathrm{HH}$ coefficients of the video signal were split into two parts, the initial part was deployed for hosting the watermark, and the second part was used at the receiver side for evaluating the system constraints that were necessary for watermark recognition. Furthermore, the simulation outcomes demonstrated that better robustness could be offered by the adopted model against several attacks, and the established scheme also offered low complexities.

\section{Proposed Video Watermarking Framework}

Figure 1 shows the framework of the adopted model. The video watermarking framework includes three stages: (i) Optimal frame prediction, (ii) Watermark embedding process, and (iii) Watermark extraction process. In the proposed model, optimal frame prediction is carried out using the DBN framework. Consider the videos, $V_{1}, V_{2}, V_{3} \ldots \ldots V_{N}$, where $N$ denotes the total number of videos. A video comprises several frames in which the embedding process can be carried out. The selection of optimal frames that is better for embedding purposes is not at all easy to define; hence, this paper uses GA, which selects frames with high PSNR. Let $f_{n}: n=1,2, . . M$ be the frames contained in the videos, and $M$ indicates the total number of frames. In the proposed work, 65 frames are optimally selected from each video. Consequently, a data library is formed from the videos, $V_{1}, V_{2}, V_{3} \ldots \ldots V_{N}$, where, for every 65 frames, the GLCM features and labels are determined. The frames are assigned with a label of one or zero, where label one denotes the frame with better PSNR, and label zero denotes the frame with reduced PSNR. This data library is then trained to the DBN framework, from which the optimal frames can be predicted at the testing phase. Furthermore, the watermark embedding process, and watermark extraction process are carried out by which the image can be embedded with the optimally selected frames. 


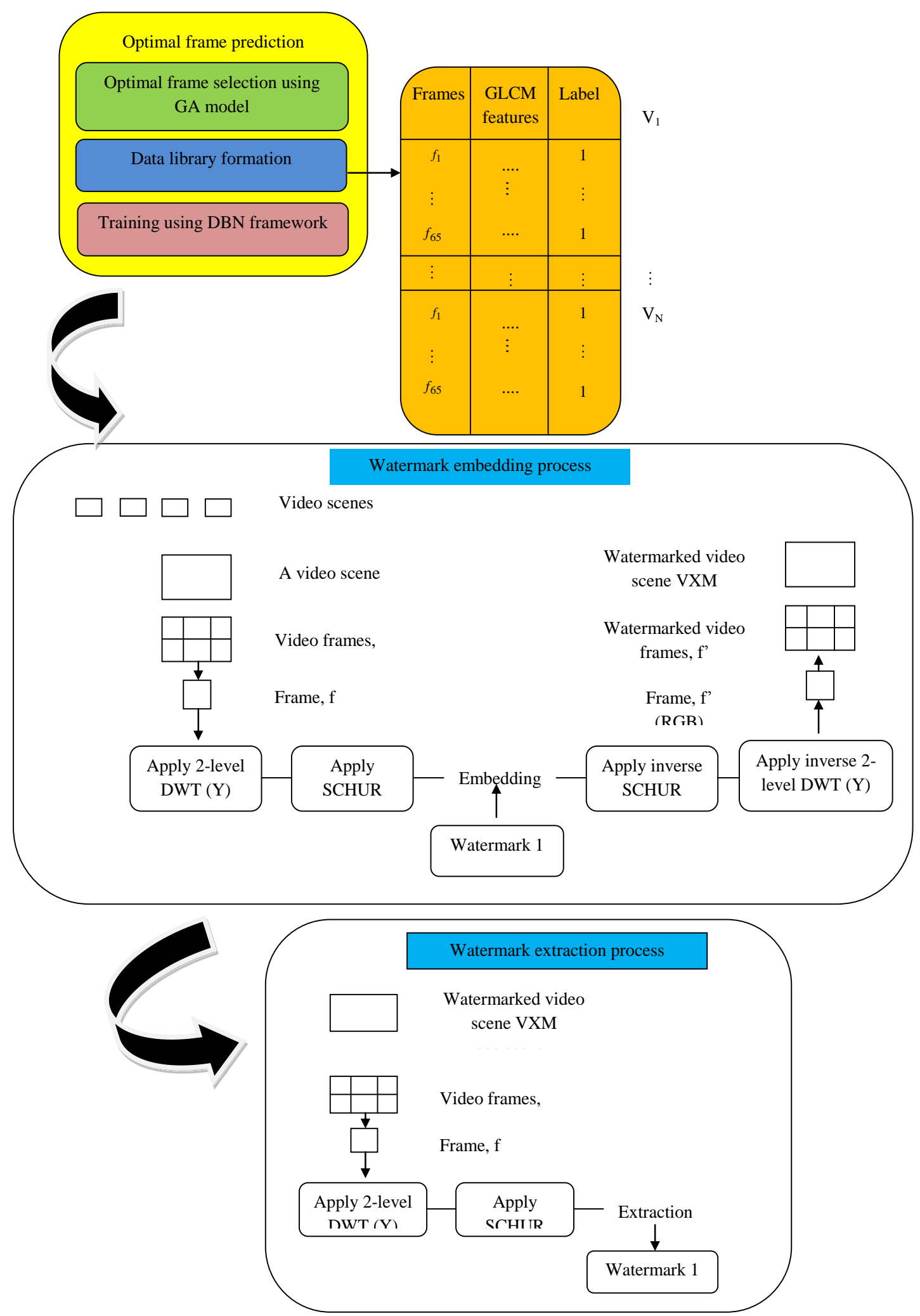

Fig. 1. Overall depiction of the adopted model. 


\section{A. Solution Encoding and Objective Function}

The solution given to GA is frames of each video, and as the work considers 65 frames as optimal, the chromosome length is 65 . The frames are assigned with a label of one or zero, where label one denotes the frame can be selected for embedding process and label zero denotes the frame cannot be embedded. Figure 2 demonstrates solution encoding. Furthermore, the objective function that decides the optimal selection of frames is determined in Eq. (1).

$$
R=\operatorname{Max}(P S N R)
$$

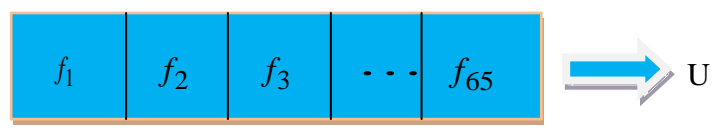

Fig. 2. Solution encoding.

\section{B. Genetic Algorithm}

The optimal frame selection is carried out by the GA algorithm. The traditional GA [21] comprises crossover, mutation, genotypephenotype mapping, fitness, selection and termination.

Crossover: This process involves mixing the genetic elements of more than one solution. All species in the world have two parents, but in certain cases, the sex of the male and female cannot be identified, and hence, such species may have one parent. In the GA model, the number of crossover operators can be more than 2 parents. Numerous species adopt several resources for choosing the right partners and attracting their partners.

Mutation: Mutation is the next level of development in the GA This stage is dependent on random variations. The efficacy of these variations is termed the mutation rate, which is also known as step size. Mutation operators are used for reachability, unbiasedness and scalability purposes.
Genotype-phenotype mapping: The population of offspring must be computed after the crossover and mutation process. Every candidate solution is chosen such that the optimization crisis is resolved. Depending on chromosome mapping, it is necessary to transfer the genotype to the original solution, which is known as phenotype.

Fitness: During the fitness computation, the phenotype of a solution is evaluated based on a fitness function, which computes the optimality of the solutions, and the GA is formed. A fitness value is allocated for every solution based on whether it is closer to the optimal solution.

Selection: To permit convergence in the optimal direction, the offspring solutions are ranked as parents in the novel parental population. More offspring solutions are created, and the best solutions are chosen to attain better growth. This selection procedure is dependent on the population of fitness values.

Termination: Termination is attained when the major evolutionary loop terminates. The GA is iterated for a few generations, which are determined earlier. The time and cost of the fitness function calculations may limit the length of the optimization process. If no further improvements are obtained by the fitness function, the algorithm is terminated.

\section{Data Library Construction}

As the main contribution, a data library is constructed with the determined optimal frames by extracting the GLCM features; GLCM features are determined as per the concept described in this section. The diagrammatic representation of the data library with the defined columns is shown in Fig. 1. The brief portrayal of the GLCM ${ }^{[22]}$ features is described in this section. 
GLCM features:

1. Energy is given by $E=\sum_{\tilde{i}} \sum_{j} g_{a \tilde{i} j}^{2}$, where $g_{a \tilde{i} j}$ is the $(\tilde{i}, j)^{t h}$ entry in GLCM

2. Entropy is given by

Ent $=-\sum_{\tilde{i}} \sum_{j} g_{a \tilde{i} j} \log _{2} g_{a \tilde{i} j}$

3. Contrast is given by

Con $=\sum_{\tilde{i}} \sum_{j}(\tilde{i}-j)^{2} g_{a \tilde{i} j}$

4. Variance is given by $V=\sum_{\tilde{i}} \sum_{j}(\tilde{i}-\mu)^{2} g_{a \tilde{i} j}$, where $\mu$ is the mean of $g_{a \tilde{i} j}$

5. Homogeneity is given by $H=\sum_{\tilde{i}} \sum_{j} \frac{1}{1+(\tilde{i}-j)^{2}} g_{a \tilde{i} j}$

6. Correlation is given by $C=\frac{\sum_{\tilde{i}} \sum_{j}(\tilde{i} j) g_{a \tilde{i} j}-\mu_{x} \mu_{y}}{\sigma_{x} \sigma_{y}}$, where $\mu_{x,} \mu_{y}$, $\sigma_{x}, \sigma_{y}$ are the mean and standard deviations of $g_{a_{x}}, g_{a_{y}}$

7. Sum average is given by $S A=\sum_{\tilde{i}=2}^{2 N_{g}} \widetilde{\ddot{i}} g_{a_{x+y}}(\tilde{i})$, where $N_{g}$ denotes the distinct gray levels in the image.

8. Sum entropy is given by

$$
S E=\sum_{\tilde{i}=2}^{2 N_{g}} g_{a_{x+y}}(\tilde{i}) \log \left\{g_{a_{x+y}}(\tilde{i})\right\}
$$

9. Sum variance is given by

$$
S V=\sum_{\tilde{i}=2}^{2 N_{g}}(\tilde{i}-S A)^{2} g_{a_{x+y}}(\tilde{i})
$$

10. Difference variance is given by $D V=$ variance in $g_{a_{x-y}}$
11. Difference entropy is given by

$$
D E=\sum_{i=0}^{N_{g-1}} g_{a_{x-y}}(\tilde{i}) \log \left\{g_{a_{x-y}}(\tilde{i})\right\}
$$

12. The maximum correlation coefficient $\left(2^{\text {nd }} \text { largest eigenvalue of } Q\right)^{0.5}$ is given by $M C C=\sum_{k} \frac{g_{a}(\tilde{i}, k) g_{a}(j, k)}{g_{a_{x}}(\tilde{i}) g_{a_{y}}(k)}$

13. The information measures of correlation 1 are given by $I M C 1=\frac{H X Y-H X Y 1}{\max \{H X, H Y\}}$ Information measures of correlation 2 are given by

$$
\begin{gathered}
I M C 2=\sqrt{(1-\exp [-2.0[H X Y 2-H X Y]]),} \\
\text { where } H X Y=-\sum_{\tilde{i}} \sum_{j} g_{a \tilde{i} j} \log _{2} g_{a \tilde{i} j} \\
H X Y 1=-\sum_{\tilde{i}} \sum_{j} g_{a \tilde{i} j} \log _{2}\left\{g_{a_{X}}(\tilde{i}) g_{a y}(j)\right\} \\
H X Y 2=-\sum_{\tilde{i}} \sum_{j} g_{a_{x}}(\tilde{i}) g_{a y}(j) \log _{2}\left\{g_{a_{x}}(\tilde{i}) g_{a y}(j)\right\}
\end{gathered}
$$

\section{Optimal Frame Prediction Using DBN}

After the data library is constructed, it is trained in the DBN (training process), from which the optimal frames for the embedding process can be predicted (testing process). The DBN ${ }^{[20]}$ framework is a well-known intelligent approach that comprises numerous layers, visible neurons and hidden neurons form the output layer. The output $\overline{P O}$ in Eq. (3) is based on probability function $\bar{P}_{q}(\zeta)$. Here, $t^{P}$ denotes the pseudotemperature. The DBN model is specified in Eq. (4).

$$
\begin{aligned}
& \bar{P}_{q}(\zeta)=\frac{1}{1+e^{\frac{-\zeta}{t^{p}}}} \\
& \overline{P O}=\left\{\begin{array}{ll}
1 & \text { with } 1-\bar{P}_{q}(\zeta) \\
0 & \text { with } \bar{P}_{q}(\zeta)
\end{array}\right\}
\end{aligned}
$$

$$
\lim _{t^{P} \rightarrow 0^{+}} \bar{P}_{q}(\zeta)=\lim _{t^{P} \rightarrow 0^{+}} \frac{1}{1+e^{\frac{-\zeta}{t^{P}}}}=\left\{\begin{array}{lll}
0 & \text { for } & \zeta<0 \\
\frac{1}{2} & \text { for } & \zeta=0 \\
1 & \text { for } & \zeta>0
\end{array}\right.
$$


The mathematical design reveals the Boltzmann machine energy for the formation of binary state $b i$ or neuron, which is shown in Eq. (5) and Eq. (6), in which $L_{a, l}$ denotes the weights between neurons and $\theta_{a}$ refers to the biases.

$$
\begin{aligned}
& E N(b i)=-\sum_{a<l} b i_{a} L_{a, l}-\sum_{a} \theta_{a} b i_{a} \\
& \Delta E N\left(b i_{a}\right)=\sum_{l} b i_{a} L_{a, l}+\theta_{a}
\end{aligned}
$$

The development of energy with respect to the joint composition of hidden and visible neurons $(x, y)$ is portrayed in Eq. (7), Eq. (8) and Eq. (9). Here, $x_{a}$ and $y_{a}$ denote the binary state of $a$, the visible unit, and hidden unit $l$. $k_{a}$ and $C_{l}$ indicate the biases, and $L_{a, l}$ specifies the weight among them.

$$
\begin{aligned}
& E N(x, y)=-\sum_{(a, l)} L_{a, l} x_{a} y_{l}-\sum_{a} k_{a} x_{a}-\sum_{l} C_{l} y_{a} \\
& \Delta E N\left(x_{a}, \bar{y}\right)=\sum_{l} L_{a l} y_{l}+k_{a} \\
& \Delta E N\left(\vec{x}, y_{a}\right)=\sum_{l} L_{a l} x_{a}+C_{l}
\end{aligned}
$$

RBM training can achieve the distributed probabilities, and the resultant weight allocation is given by Eq. (10).

$$
\left.\hat{L}_{\hat{G}}\right)=\max _{\hat{L}} \prod_{\vec{x} \in N} c(\vec{x})
$$

For the hidden and visible vectors pair $(\vec{x}, \overrightarrow{h i})$, the probability distributed RBM model is specified in Eq. (11), in which $P R^{F}$ denotes the partition function as given in Eq. (12).

$$
\begin{aligned}
& c(\vec{x}, \overrightarrow{h i})=\frac{1}{P R^{F}} e^{-E N(\vec{x}, \vec{y})} \\
& P R^{F}=\sum_{\vec{x}, \vec{y}} e^{-E N(\vec{x}, \vec{y})}
\end{aligned}
$$

Prior to the learning process of the MLP model, assume $\left(K^{\hat{G}}, W^{\hat{G}}\right)$ training patterns, in which $\hat{G}$ denotes the number of training patterns, $1 \leq \hat{G} \leq \bar{P}, \quad K^{\hat{G}}$ and $W^{\hat{G}}$ denote the input vector together with the required output vectors, respectively. Eq. (13) demonstrates every neuron error in $l$ of the output layer.

$$
e_{l}^{\hat{G}}=K^{\hat{G}}-W^{\hat{G}}
$$

Therefore, Eq. (13) describes the squared error of the $\hat{M}$ pattern subsequent to MSE, as in Eq. (14) and Eq. (15), in which $\tilde{P}$ denotes the number of training patterns.

$$
\begin{aligned}
& S E_{\hat{G}}^{\text {mean }}=\frac{1}{\tilde{o}_{y}} \sum_{l=1}^{\tilde{o}_{y}}\left(e_{l}^{\hat{G}}\right)^{2}=\frac{1}{\tilde{o}_{y}} \sum_{l=1}^{\tilde{o}_{y}}\left(K^{\hat{G}}-W^{\hat{G}}\right)^{2} \\
& S E_{\text {avg }}=\frac{1}{\tilde{P}} S E_{\hat{G}}^{\text {mean }}
\end{aligned}
$$

\section{Video Watermark Embedding and Watermark Extraction Process}

\section{A. Watermark Embedding Process}

The embedding process ${ }^{[23]}$ is as follows:

Step 1: Video clip $V$ is given as input.

Step 2: The video clips are split into video scenes, $V_{s_{i}}$

Step 3: The frames are processed for every video by means of DWT and Schur as given in steps 4-11.

Step 4: Each video frame is transformed (optimally selected frames), $f$ to YUV color matrix form from RGB form.

Step 5: 2-level DWT is computed for the Y matrix in every $f$, which produces 7 DWT subbands such as, $\left[l l_{1}, l l_{2}, h l_{2}, l h_{2}, h h_{2}, l h_{1}, h h_{1}\right]$.

Step 6: The "Schur operator" is deployed on the $h l_{2}$ subband, which decomposes the coefficient matrix of the subband into two independent matrices $h l_{2}=\left(u_{h l_{2}}, s_{h l_{2}}\right)$

Step 7: The watermark image is rescaled, and thus, the watermark size matches the $h l_{2}$ subband size, which can be exploited for embedding. 
Step 8: The watermark's binary bits $W_{V_{s_{i}}}$ are embedded into the diagonal matrix of $s_{h_{2}}$ by replacing the watermark bit $W_{i}$ with the $8^{\text {th }}$ LSB bit of $s_{h l_{2}},(i, i)$ as shown in Eq. (16).

$$
\operatorname{LSB}\left(s_{h l_{2}},(i, i)\right)=W_{S_{s_{i}}}
$$

Step 9: The inverse Schur operator is deployed on the updated $s^{\prime}{ }_{h l_{2}}$ matrix to attain an updated coefficient matrix $h l_{2}^{\prime}$. The inverse Schur function is given by Eq. (17).

$$
\left(u_{h l_{2}} \times s_{h l_{2}} \times u_{h l_{2}}^{T}\right)
$$

Step 10: The inverse DWT is deployed on $h l_{2}^{\prime}$. This function generates the final watermarked video frame $f^{\prime}$.

Step 11: $f^{\prime}$ is transformed to the RGB color matrix from the YUV format.

Step 12: $f$ is rebuilt to $f^{\prime}$.

Step 13: The watermarked scenes are rebuilt to attain the final watermarked VXM.

\section{E. Watermark extraction process}

The watermark image ${ }^{[23]}$ can be directly extracted from the watermarked video frames from the LSBs. The process of watermark extraction is as follows:

Step 1: Video clip $V^{\prime}$ is given as input.

Step 2: $V^{\prime}$ is split into video scenes, $V_{s_{i}}^{\prime}$

Step 3: The frame is processed for every watermarked video scene by means of Schur and DWT as given in steps 4-7.

Step 4: Each video frame transforms $f^{\prime}$ to YUV from the RGB color matrix form.

Step 5: 2-level DWT is computed for $f^{\prime}$, which produces 7 DWT subbands such as, $\left[w l_{1}, w l l_{2}, w h l_{2}, w l h_{2}, w h h_{2}, w l h_{1}, w h h_{1}\right]$.
Step 6: The "Schur operator" is deployed on all $w h l_{2}$ subbands, which decomposes the coefficient matrix of the subband into two independent matrices as given by Eq. (18).

$$
w h l_{2}=\left(u_{w h l_{2}}, s_{w h l_{2}}\right)
$$

Step 7: The embedded watermark is embedded from the diagonal matrix of $s_{w h l_{2}}$ as shown in Eq. (19).

$$
L S B\left(s_{h l_{2}},(i, i)\right)=W_{V_{s_{i}}}(i)
$$

Step 8: A watermark image $W_{V_{s_{i}}}$ is constructed by cascading the bits of watermarks extracted from the entire frame.

Step 9: A similar process is continued for all the video scenes.

\section{Results and Discussion}

\section{A. Simulation Procedure}

The implemented GA technique for video watermarking was simulated in MATLAB 2018 a, and the outcomes were attained. The experiment was performed by analyzing MSE, PSNR and SDME measures with respect to varying embedded frames. Furthermore, statistical analysis was conducted with respect to all the measures, and the improvement of the adopted model was proven over the conventional PSO ${ }^{[24]}$ model. The sample images of the existing and proposed model are shown in Fig. 3.

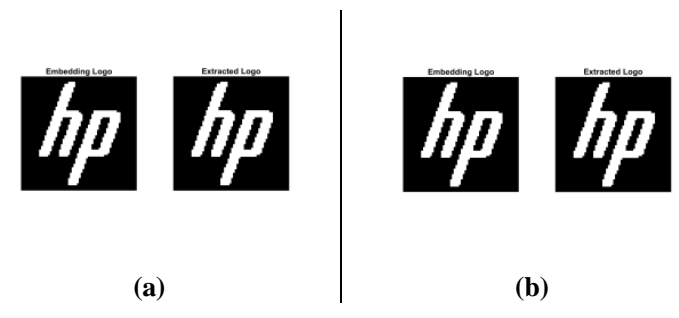

Fig. 3. Sample video watermarked image of the (a) PSO (b) GA.

\section{B. Error Performance}

The error performance of the proposed GA scheme is described in this section. Here, 
from Fig. 4(a), the attained graph on the MSE shows that the presented method offers a reduced error performance over the PSO model with respect to varied embedded frames. Here, the adopted scheme is $23.53 \%$ superior to the existing model at 60 embedded frames Additionally, as shown in Fig. 4(b), a better PSNR was achieved by the implemented model over the existing PSO scheme, which is $4.54 \%$ better than the existing model at 50 embedded frames. Furthermore, from Fig. 4(c), the SDME measure was higher for the presented model, which is $2.59 \%$ superior to the PSO model with respect to 55 embedded frames. Therefore, the superiority of the presented GA approach has been validated effectively.
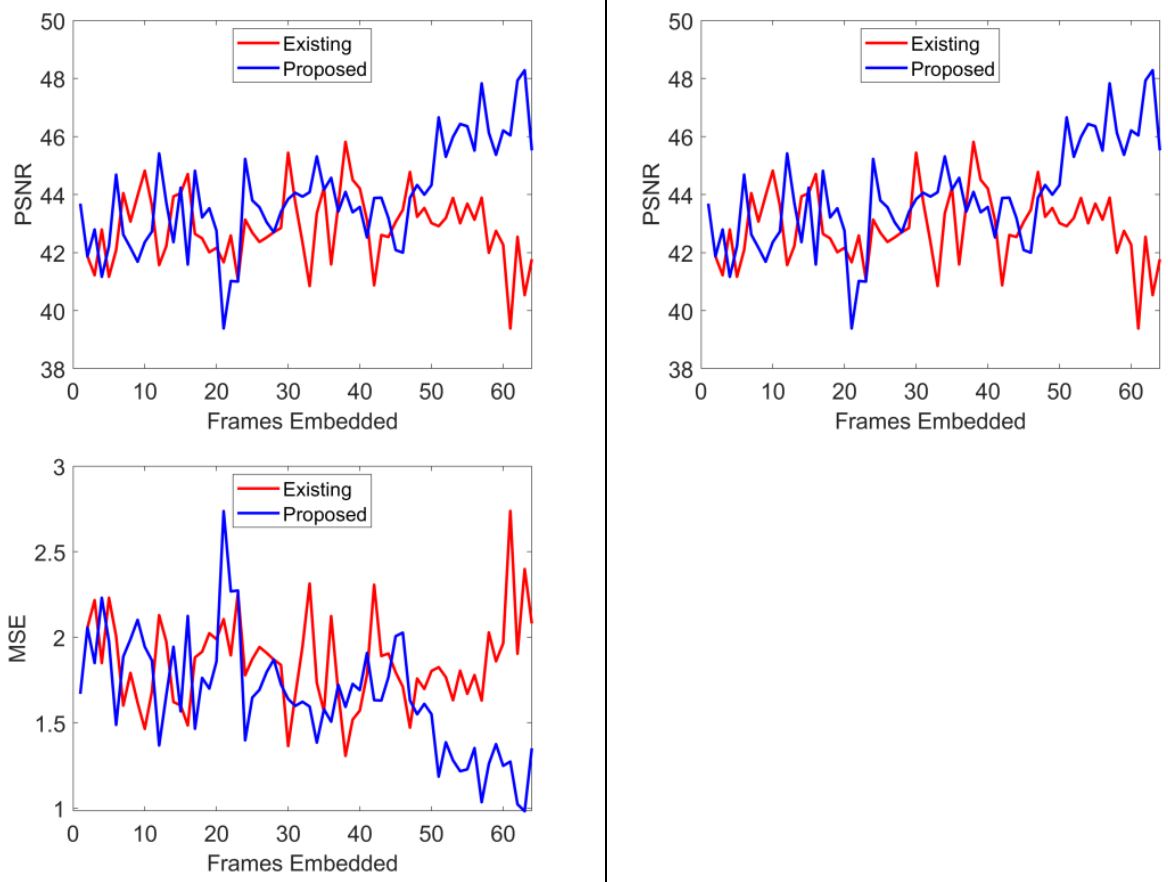

(a)

(b)

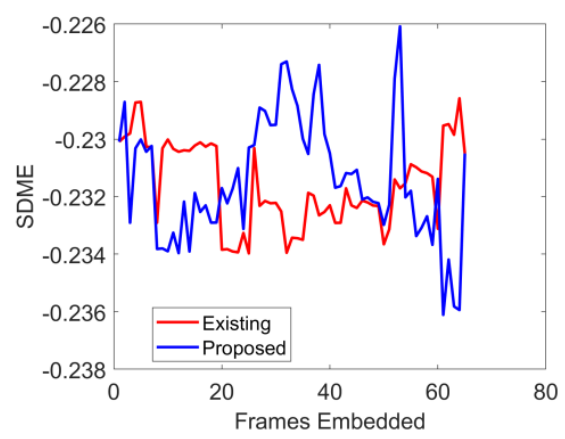

(c)

Fig. 4. Performance analysis of the proposed model over the conventional model for (a) MSE (b) PSNR (c) SDME. 


\section{Statistical Performance}

Since the metaheuristic algorithms are stochastic in nature, the algorithms were executed five times, and the results were obtained. The statistical performance of the adopted GA scheme is portrayed in Tables 13. From Table 1, the best, mean, median and standard deviation of the presented GA model are $0.3 \%, 2.13 \%, 0.48 \%$, and $4.39 \%$ better, respectively, than the traditional scheme. Similarly, from Table 2, the best, mean, median and standard deviation of the proposed GA model are $7.65 \%, 0.32 \%, 0.59 \%$ and $2.35 \%$ superior, respectively, to the traditional PSO scheme. Additionally, from Table 3, the best, mean, median and standard deviation of the implemented GA scheme are $16.69 \%$, $7.14 \%, \quad 16.01 \%$ and $8.63 \%$ superior, respectively, to the conventional PSO scheme. Thus, the effectiveness of the proposed GA algorithm has been proven by the achieved results.

Table 1. Statistical analysis of MSE performance for the proposed model over the conventional model.

\begin{tabular}{|l|r|r|}
\hline Measures & PSO [24] & \multicolumn{1}{l|}{ GA } \\
\hline Best & $7.51 \times 10^{1}$ & $7.49 \times 10^{1}$ \\
\hline Worst & 2.30 & 2.30 \\
\hline Mean & 1.30 & 1.27 \\
\hline Median & 1.16 & 1.15 \\
\hline Std-Dev & $5.23 \times 10^{1}$ & $5.00 \times 10^{1}$ \\
\hline
\end{tabular}

Table 2. Statistical analysis of PSNR performance for the proposed model over conventional models.

\begin{tabular}{|l|r|r|}
\hline Measures & PSO [24] & GA \\
\hline Best & $5.06 \times 10^{1}$ & $5.07 \times 10^{1}$ \\
\hline Worst & $4.09 \times 10^{1}$ & $4.09 \times 10^{1}$ \\
\hline Mean & $4.65 \times 10^{1}$ & $4.66 \times 10^{1}$ \\
\hline Median & $4.70 \times 10^{1}$ & $4.73 \times 10^{1}$ \\
\hline Std-Dev & 3.33 & 3.41 \\
\hline
\end{tabular}

Table 3. Statistical analysis of sdme performance for the proposed model over conventional models.

\begin{tabular}{|l|c|c|}
\hline Measures & PSO [24] & GA \\
\hline Best & $1.44 \times 10^{-1}$ & $1.23 \times 10^{-1}$ \\
\hline Worst & $-4.19 \times 10^{-1}$ & $-4.19 \times 10^{-1}$ \\
\hline Mean & $-9.87 \times 10^{-2}$ & $-9.16 \times 10^{-2}$ \\
\hline Median & $-1.03 \times 10^{-1}$ & $-8.67 \times 10^{-2}$ \\
\hline Std-Dev & $1.74 \times 10^{-1}$ & $1.59 \times 10^{-1}$ \\
\hline
\end{tabular}

\section{Conclusion}

This paper presented a video watermarking framework that comprises optimal video frame prediction and a watermark embedding process and watermark extraction processes. Here, the optimal frame prediction was performed using the DBN framework. First, randomly chosen frames from each video were provided as input to the GA model, which optimally selected the frames such that the PSNR should be high. The frames were assigned with a label of one or zero, where label one denoted the frame with better PSNR and label zero denoted the frame with reduced PSNR. In addition, a data library was created from the outcomes, in which every video frame was determined with its GLCM features and labels. This data library was further trained in the DBN framework, and thus, the optimal frames were predicted. In addition, watermark embedding and watermark extraction were performed by which the image could be embedded within the optimally selected frames. From the analysis, the adopted scheme was $23.53 \%$ superior to the PSO model at 60 embedded frames. Additionally, the PSNR achieved by the implemented model over the existing PSO scheme was $4.54 \%$ better than the existing model at 50 embedded frames. Thus, the efficiency of the presented approach was proven from the simulation outcomes.

\section{Declaration}

I, Tthe undersigned declare that this manuscript is original, has not been published before and is not currently being considered for publication elsewhere.

\section{Conflict of Interest}

The author declared that there is no conflict of interest.

\section{Availability of data and material}

Not applicable 


\section{Competing interests}

\author{
Not applicable
}

\section{Funding}

Not applicable

\section{Author's contributions}

Conceived and designed the analysis, collected data, performed the analysis, wrote the paper

\section{Acknowledgments}

The authors would like to thank Majid Alotaibi for lab assistance during the research.

\section{References}

[1] Sake, A. and Ramashri, T. (2018) "Bi-orthogonal Wavelet Transform Based Video Watermarking Using Optimization Techniques", Materials Today, 5(1) Part 1: 1470-1477.

[2] Sahu, N. and Sur, A. (2017) "SIFT based video watermarking resistant to temporal scaling", Journal of Visual Communication and Image Representation, 45(May): 77-86.

[3] Dutta, T. and Gupta, H. P. (2016) "A robust watermarking framework for High Efficiency Video Coding (HEVC) - Encoded video with blind extraction process", Journal of Visual Communication and Image Representation, 38(July): 29-44.

[4] Rasti, P., Samiei, S., Agoyi, M., Escalera, S. and Anbarjafari, G. (2016) "Robust non-blind color video watermarking using QR decomposition and entropy analysis", Journal of Visual Communication and Image Representation, 38(July): 838-847.

[5] Madine, F., Akhaee, M. A. and Zarmehi, N. (2018) "A multiplicative video watermarking robust to H.264/AVC compression standard", Signal Processing: Image Communication, 68(October): 229-240.

[6] Roy, S. and Pal, A. K. (2017) "A blind DCT based color watermarking algorithm for embedding multiple watermarks", AEU - International Journal of Electronics and Communications, 72(February): 149-161.

[7] Liu, X., Zhao, R., Li, F., Liao, S. and Zou, B. (2017) "Novel robust zero-watermarking scheme for digital rights management of 3D videos", Signal Processing: Image Communication, 54(May): 140-151.

[8] Ansari, A., Hong, S., Saavedra, G., Javidi, B. and Martinez-Corral, M. (2018) "Ownership protection of plenoptic images by robust and reversible watermarking”, Optics and Lasers in Engineering, 107(August): 325-334.
[9] Liansheng, S., Yin, C., Ailing, T. and Asundi, A. K. (2018) "An optical watermarking scheme with two-layer framework based on computational ghost imaging", Optics and Lasers in Engineering, 107(August): 38-45.

[10] Pan, W., Bouslimi, D., Karasad, M., Cozic, M. and Coatrieux, G. (2018) "Imperceptible reversible watermarking of radiographic images based on quantum noise masking", Computer Methods and Programs in Biomedicine, 160(July): 119-128.

[11] Zheng, Z., Saxena, N., Mishra, K. K. and Sangaiah, A. K. (2018) "Guided dynamic particle swarm optimization for optimizing digital image watermarking in industry applications", Future Generation Computer Systems, 88 (November): 92-106.

[12] Liu, Y., Tang, S., Liu, R., Zhang, L. and Ma, Z. (2018) "Secure and robust digital image watermarking scheme using logistic and RSA encryption", Expert Systems with Applications, 97(1 May): 95-105.

[13] Li, Hui-F., Chang, N. and Chen, Xiao-M. (2010) “A study on image digital watermarking based on wavelet transform", The Journal of China Universities of Posts and Telecommunications, 17(July), Supplement 1: 122126.

[14] Sk, A. and Masilamani, V. (2018) "A novel digital watermarking scheme for data authentication and copyright protection in 5G networks", Computers \& Electrical Engineering, 9 (March).

[15] Vaidya, S. P. and Mouli, P. V. S. S. R. C. (2015) "Adaptive Digital Watermarking for Copyright Protection of Digital Images in Wavelet Domain", Procedia Computer Science, 58: 233-240.

[16] Loukhaoukha, K. (2013) "Comments on "A digital watermarking scheme based on singular value decomposition and tiny genetic algorithm", Digital Signal Processing, 23(4) July: 1334.

[17] Cedillo-Hernandez, A., Cedillo-Hernandez, M., Miyatake, M. N. and Meana, H. P. (2018) "A spatiotemporal saliency-modulated JND profile applied to video watermarking", Journal of Visual Communication and Image Representation, 52(April): 106-117.

[18] Lv, T., Zhang, He-Y. and Yan, Chun-H. (2018) "Double mode surveillance system based on remote audio/video signals acquisition", Applied Acoustics, 129(1 January): 316-321.

[19] Loganathan, A. and Kaliyaperumal, G. (2016) "An adaptive HVS based video watermarking scheme for multiple watermarks using BAM neural networks and fuzzy inference system", Expert Systems with Applications, 63(30 November): 412-434.

[20] Wang, H.Z., Wang, G.B., Li, G.Q., Peng, J.C. and Liu, Y.T. (2016) "Deep belief network based deterministic 
and probabilistic wind speed forecasting approach", Applied Energy, 182: 80-93,.

[21] Vrionis, T. D., Koutiva, X. I. and Vovos, N. A. (2014) "A Genetic Algorithm-Based Low Voltage Ride-Through Control Strategy for Grid Connected Doubly Fed Induction Wind Generators", IEEE Transactions on Power Systems, 29(3) May.

[22] Gadkari, D. (2004) "Image Quality Analysis using GLCM". https://stars.library.ucf.edu/cgi/viewcontent.cgi?article=1 $186 \&$ context=etd, University of Central Florida.

[23] Rajab, L., Al-Khatib, T. and Al-Haj, A. (2015) "A Blind DWT-SCHUR Based Digital Video Watermarking Technique", Journal of Software Engineering and Applications, 8: 224-233.

[24] Zhang, J. and Xia, P. (2017) "An improved PSO algorithm for parameter identification of nonlinear dynamic hysteretic models", Journal of Sound and Vibration, 389(17 February): 153-167. 
العلامة المائية لحماية حقوق الطبع والنشر لمقاطع الفيديو بالاعتماد على اختيار الإطار الوراثي للمقطع

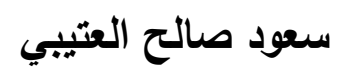

قسم نظم المعلومات، كلبة الحاسب الآلي ونظم المعلومات، جامعة أم القرى، مكة المكرمة، المدلكة العربية السعودية

ssotaibi@uqu.edu.sa

المستخلص. في الآونة الأخيرة، اهتم الباحثون بشكل ملحوظ بمجال العلامة المائية لمقاطع الفيديو • فقد تم تتفيذ العديد من التطبيقات لهذه التقنية في مجموعة منتوعة من المجالات. لذلك، لهات تهدف هذه الورقة إلى صباغة إطار عمل جديد للعلامات المائية للفيديو، يتضمن ثلاث مراحل: (1) التتبؤ الأمثل لإطار مقطع الفيديو، (r) مرحلة تضمين العلامة المائية، (r) مرحلة استخراج العلامة المائية. ففي النموذج المقترح، يتم تتفيذ تتبؤ الإطار الأمتل باستخدام مبدأ التعلم العميق المتمنل في خوارزميات (DBN) (deep belief network). حيث تُستخدم الإطارات التي يتم اختيارها عشوائًاً من كل مقطع فيديو كمدخل لنموذج الخوارزمية الجينية ( genetic

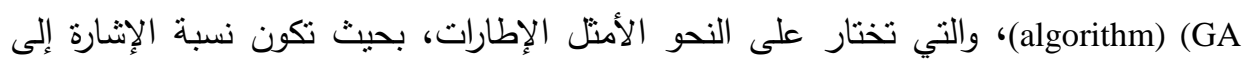

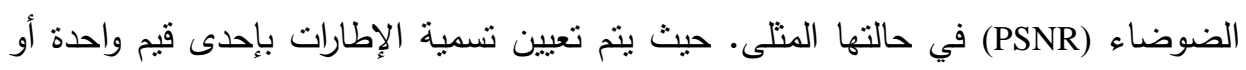

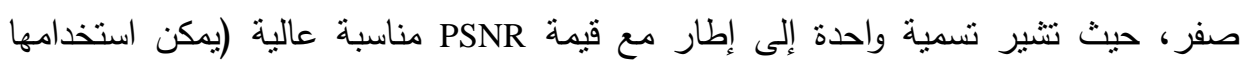
خلال عملية التضمين) وتسمية الصفر نتير إلى الإطار مع قيمة PSNR منخفضة (لا يمكن

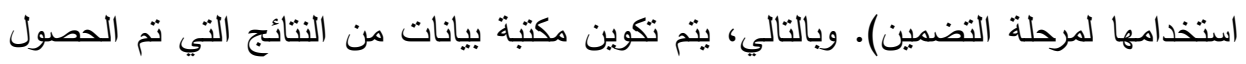
عليها، حيث يتم تحديد كل إطار فيديو بمصفوفة المميزات والعناصر الخاصة بها، والتي نم تكوينها خلال المرحلة السابقة. وباستخدام خوارزميات التعلم العميق (deep belief network)

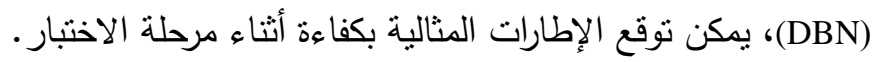
الكلمات المفتاحية: العلامة المائية لمقاطع الفيديو، الخوارزمية الجينية، مصفوفة المميزات، التعلم العميق، قياسات الخطأ. 
